were included. Data were collected from computerised clinical notes for analysis.

Results Of 57 patients (39 male and mean age 80), 47 $(82.5 \%)$ patients died during admission or within 5 days of discharge. $10(17.5 \%)$ patients survived to discharge. Non-survivors had a median CFS of 4; IQR 3-5, as did survivors (median CFS 4; IQR 3-4). Non-survivors had a median of 3 (IQR 2-4) significant comorbidities, and survivors had 2.5 (IQR 2-3.8). Median $\mathrm{P} / \mathrm{F} \quad(\mathrm{PaO} 2 / \mathrm{FiO} 2)$ ratio prior to commencing CPAP was 10.5 (IQR 8.4-12.6) for non-survivors and 14.4 (IQR 12.9-18.8) for survivors. The odds of death were 6.8 ( $\mathrm{p}$ value $<0.01$ ) in those with a severe $\mathrm{P} / \mathrm{F}$ ratio $(<13.3)$.

Conclusion This evidence indicates that CPAP used in patients aged 75 and over, particularly those with a severe $\mathrm{P} / \mathrm{F}$ ratio prior to commencing CPAP, does not improve mortality. These findings can inform future decision-making and CPAP protocol development to potentially limit its use in this group. Further study of less invasive alternative management options, such as nasal high flow oxygen, is recommended.

\section{P185 EXPERIENCE OF USING HIGH FLOW NASAL OXYGEN FIRST LINE TO TREAT HYPOXEMIC RESPIRATORY FAILURE DUE TO COVID 19 IN PATIENTS IN WHOM CRITICAL CARE ADMISSION WAS FELT TO BE NOT OF BENEFIT ON A RESPIRATORY SUPPORT UNIT (RSU) FROM OCTOBER 2020 TO MARCH 2021}

DA Tarpey, S Woods, S Jain. Wirral University Teaching Hospital, Merseyside, UK

\subsection{6/thorax-2021-BTSabstracts.294}

Introduction The efficacy of High Flow Nasal Oxygen in severe COVID 19 pneumonia is uncertain with conflicting guidance. Wirral University Teaching Hospital has used HFNO outside of critical care for some time and so in the abscnce of clear evidence we used HFNO as first line for patients with confirmed severe COVID 19 pneumonia (defined as requiring 60\% $\mathrm{FiO} 2$ despite awake proning and appropriate treatment) in whom Critical care admission was felt to be inappropriate.

Setting Enhanced respiratory support (HFNO or CPAP) was delivered on a newly developed RSU consisting of 8 negative pressure side rooms and a 5 bed cohort area. There was a daily respiratory and critical care MDT in which severely ill COVID 19 patients were discussed and escalation plans were made. Outside of this, the decision to use HFNO was made by an on call respiratory consultant and there was daily consultant review. Only patients were placed on HFNO first line or CPAP if failing/were felt more likely to benefit from CPAP.

Results 69 patients received Respiratory support (median age 72) and had had been an IP for a median of 4.2 days prior to starting HFNO. Overall $31(45 \%)$ of such patients survived to hospital discharge and received a mean FiO2 of $86 \%$. Overall LOS was on average 15 days with 6 days spent on respiratory support. Markers of poor outcome include use of CPAP (survival 4/15, 27\%), acute significant impairment of 2 organs (survival 4/14 28\%), being over 75 (36\% survival) and needing more than 90\% FiO2 (28\% survival). There was no increase in staff sickness, nosocomial infections or reported patient safety incidents. The overall survival rate compares well to ICNARC data for this cohort of patients and the previous pre pandemic local data for HFNO use in respiratory patients ( $45 \%$ from October 2020 vs $37 \%$ pre March 2020)

Conclusion HFNO appears to be a safe and effective treatment for patients with severe COVID 19 pneumonia who would not benefit from critical care support when cohorted with 24 hour senior respiratory support. The risk of nosocomial transmission is low and they can be safely managed in large negative pressure with no significant patient safety issues.

\section{P186 SINGLE CENTRE EXPERIENCE OF TOCILIZUMAB IN COVID 19 PNEUMONIA}

Y Maung Maung Myint, R Goodka, M Mehta, S Ananth, H Ghani, R Vancheeswaran. West Hertfordshire Hospitals NHS Trust, Watford, UK

\subsection{6/thorax-2021-BTSabstracts.295}

Introduction Tocilizumab is an interleukin-6 (IL-6) receptor blocker which blocks the IL-6 signal transduction pathway reducing inflammation. It is thought to be an effective drug for patients with severe COVID-19 pneumonia. Our study sought to evaluate the benefits of tocilizumab in patients with COVID 19 pneumonia who required hospital admission.

Methods This is a retrospective analysis of 47 patients with COVID 19 pneumonia who were admitted to a single centre, London district general hospital during January and February 2021. COVID 19 patients requiring high level of oxygen to maintain oxygen saturation above $92 \%$ with one of the following criteria (C-reactive protein above 50 , ferritin above $500 \mathrm{mg} / \mathrm{l}$, ddimer above $1000 \mathrm{mg} / \mathrm{ml}$ or LDH above $250 \mathrm{U} /$ L) were eligible to receive tocilizumab. These patients were given single dose of tocilizumab $8 \mathrm{mg} / \mathrm{kg}$ during first week of hospital admission. These patients were matched against 38 control patients with COVID 19 pneumonia with same SF (oxygen saturation/fiO2) ratio on admission (median SF ratio $=106.67)$ who received standard treatment (dexamethasone).

Abstract P186 Table 1 Clinical parameters of the COVID 19 pneumonia patients who received tocilizumab between January and February 2021

\begin{tabular}{|c|c|c|c|c|}
\hline & & $\begin{array}{l}\text { Dexamethasone } \\
\text { (Control group) }\end{array}$ & $\begin{array}{l}\text { Dexamethasone+ } \\
\text { Tocilizumab }\end{array}$ & $p$ value \\
\hline Total & & 38 & 47 & \\
\hline Age & Median [IQR] & 61.5 & 54 & \\
\hline SF ratio & Median [IQR] & 106.67 & 106.67 & \\
\hline \multirow[t]{2}{*}{ Gender } & Male & 21 & 36 & \\
\hline & Female & 17 & 11 & \\
\hline \multirow[t]{4}{*}{ Ethnicity } & British/White & 25 & 30 & \\
\hline & $\begin{array}{l}\text { Any other white } \\
\text { background }\end{array}$ & 1 & 8 & \\
\hline & Asian & 5 & 11 & \\
\hline & Others & 8 & 6 & \\
\hline \multirow[t]{4}{*}{ Outcome } & Death & 17 & 8 & 0.008 \\
\hline & $\begin{array}{l}\text { Need for NIVI } \\
\text { HFNO }\end{array}$ & 9 & 27 & 0.002 \\
\hline & ITU admission & 12 & 22 & 0.18 \\
\hline & $\begin{array}{l}\text { Need for } \\
\text { intubation }\end{array}$ & 4 & 13 & 0.06 \\
\hline $\begin{array}{l}\text { Length of } \\
\text { Hospital Stay }\end{array}$ & Median [IQR] & 6 & 12 & $<0.001$ \\
\hline
\end{tabular}


Findings 47 patients received standard treatment with tocilizumab. Total death was $44.7 \%$ in control group and $17 \%$ in tocilizumab group $(\mathrm{p}=0.008)$. $46.8 \%$ of patients in the tocilizumab group required ITU admission compared to $31.6 \%$ in the control group $(p=0.18) .27 .6 \%$ in tocilizumab needed intubation whereas $10.5 \%$ in control group $(p=0.06) .57 .5 \%$ in tocilizumab group were escalated to non invasive ventilation ( NIV) or high flow nasal oxygen (HFNO) whereas only $23.7 \%$ in control group required ventilatory support ( $\mathrm{p}$ value $=0.002)$. Further analysis of those in the ITU cohort revealed a mortality rate of $22.7 \%$ in the tocilizumab group and $58.3 \%$ in the control group . Length of hospital stay was twice in the tocilizumab group (12 days) vs control (6 days) $(\mathrm{p}<0.001)$.

Conclusion This study showed that tocilizumab may be associated with mortality benefit but no reduction in the rate of progression to intubation or need of NIV/HFNO. Further data with larger patient cohort is required to ascertain the benefits of tocilizumab in COVID 19 pneumonia.

\section{P187 CLINICAL OUTCOMES AND TREATMENT-RELATED ADVERSE EVENTS TO TOCILIZUMAB IN SARS-COV-2 ILLNESS}

S Ahmad, E Jenkinson, R Carney, T Nahu, J Quinn, A Dwarakanath. Mid Yorkshire Hospitals NHS Trust, Wakefield, UK

\subsection{6/thorax-2021-BTSabstracts. 296}

Background Severe SARS-CoV-2 is associated with release of Interleukin-6 and other pro-inflammatory cytokines that are markers of systemic inflammation and this response may cause or exacerbate lung injury leading to life-threatening disease. Tocilizumab, an IL-6 receptor antagonist licensed in certain Rheumatological disorders has shown to have beneficial effects on mortality and reduces the need for ventilator and organ support if used early. We evaluated the clinical outcomes and treatment \&ndash;related adverse events in patients who were treated with Tocilizumab.

Method A hospital treatment protocol with inclusion/exclusion criteria, dose regimen and clinical monitoring post dose and post discharge patient alert card (figure-1) was implemented. Two clinicians had to concur to the treatment and this was limited to patients needing either Acute Respiratory Care Unit (Level-1 HDU) or ICU. Electronic medical records of all patients who had Tocilizumab between January-May 2021 were reviewed. Baseline demographics, dose regimen, respiratory support at the time of treatment and adverse events were reviewed.

Results 108 patients (age; 56 $\pm 14, \mathrm{BMI} ; 32 \pm 3$, males-66\%) received Tocilizumab. The dosing regimen was weight based $(8 \mathrm{mg} / \mathrm{kg}$, maximum $800 \mathrm{mg})$ and was given within 24 hours in $79 \%$ patients and $21 \%$ within 48 hours of admission to either ARCU or ICU. Majority (95\%) received one dose and the second dose was only considered in the absence of clinical improvement. Respiratory support at the time of Tocilizumab treatment included CPAP -93\% (PEEP; 8-12), $1 \%$ nasal high flow therapy and $6 \%$ invasive ventilation including ECMO. Over a third of patients had no complications but $67 \%$ had deranged liver functions (elevated ALT) but settled with supportive measures, $1 \%$ had thrombocytopenia and $1 \%$ had reactivation of $\mathrm{TB}$ (TB Lymphadenitis). The mortality rate in patients who received Tocilizumab was

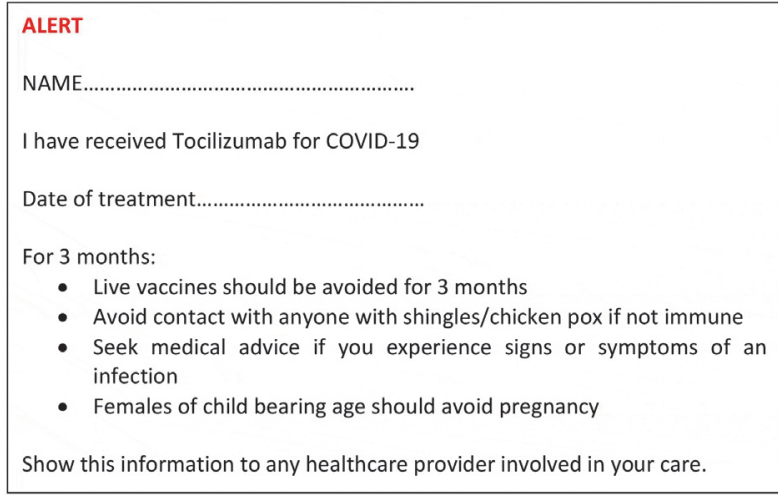

Abstract P187 Figure 1 Showing the post Tocilizumab alert card

24\% $(n=26)$. Post discharge alert card to the patients and specific discharge information to primary care were provided.

Conclusion Appropriate treatment protocol and regular monitoring are needed for patients who receive Tocilizumab in severe SARS-CoV-2 illness. Clinicians should bear in mind the high incidence of treatment-related adverse events and the lack of data about long term effects. Treatment alert cards and specific discharge advice may be beneficial.

\section{P188 TICK TOCK...WHERE AND WHEN CAN WE GIVE TOC? REVIEW OF COVID-19 PATIENTS RECEIVING TOCILIZUMAB IN A NON CRITICAL CARE SETTING}

K Aiken, M Wilson, E Keelan. Mater Hospital, Belfast, UK

\subsection{6/thorax-2021-BTSabstracts.297}

Introduction The REMAP-CAP trial demonstrated the positive effects of interleukin-6 receptor antagonists (tocilizumab and sarilumab), on mortality in COVID-19 patients managed in a critical care setting. ${ }^{1}$ Prior to this, adjuvant drug therapies such as remdesivir and dexamethasone have shown limited benefits regarding COVID-19 related mortality in patients requiring non-invasive respiratory, managed in non-critical care settings.

During the pandemic the Mater Hospital, Belfast was designed as the local COVID-19 centre. Prior to January 2021 standard ward level care included IV antibiotics, IV remdesivir, oral dexamethasone and non-invasive ventilation. Continuous positive airway pressure was used first line (commenced when $\mathrm{FiO} 2$ requirements exceeded $4 \mathrm{~L} / \mathrm{min}$ via nasal cannula). After the release of the Department of Health's position statement regarding tocilizumab for COVID-19 patients on respiratory support, a decision was taken to use tocilizumab off license in a non-critical care setting.

Our hypothesis was that COVID-19 positive patients on non-invasive ventilation who received tocilizumab in addition to standard care would have reduced hospital mortality compared with standard care alone. The REMAP-CAP trial administered tocilizumab to COVID-19 patients in a critical care setting, however we postulated that those 'less unwell' patients requiring ward level respiratory support but not 'critical care' could still benefit.

Methods Patients commenced on tocilizumab in a non-critical care setting were identified and followed up prospectively. A 